PROCEEDINGS OF THE

AMERICAN MATHEMATICAL SOCIETY

Volume 126, Number 3, March 1998, Pages 877-880

S $0002-9939(98) 04458-X$

\title{
SIMPLE QUOTIENTS OF HYPERBOLIC 3-MANIFOLD GROUPS
}

\author{
D. D. LONG AND A. W. REID \\ (Communicated by James West)
}

\begin{abstract}
We show that hyperbolic 3-manifolds have residually simple fundamental group.
\end{abstract}

\section{INTRODUCTION}

Let $G$ be a finitely generated group and $X$ a property of groups, e.g. finite, simple, $p$-group. $G$ is said to be residually $X$, if for any element $g \neq 1$, there is a group $H$ with property $X$ and a surjective homomorphism $\phi: G \rightarrow H$ such that $\phi(g) \neq 1$.

Of interest to us are residual properties of groups $\pi_{1}(M)$ where $M$ is a compact orientable 3-manifold with infinite fundamental group. Now it is well-known that if $M$ is a hyperbolic 3-manifold, that is the quotient of hyperbolic 3-space by a torsion-free Kleinian group, then $\pi_{1}(M)$ is residually finite. In this note we prove a much stronger result which seems to have been unnoticed previously. First we make a definition.

Definition 1.1. Let $M$ be a compact orientable 3-manifold with infinite fundamental group and $\rho: \pi_{1}(M) \rightarrow S L(2, \mathbb{C})$ a faithful representation whose image lies in $S L(2, \overline{\mathbb{Q}})$, where $\overline{\mathbb{Q}}$ is the algebraic closure of $\mathbb{Q}$. In this case we define $\rho$ to be an algebraic representation.

With this we can state:

Theorem 1.2. Let $M$ be a compact orientable 3-manifold such that $\pi_{1}(M)$ admits an algebraic representation. Then $\pi_{1}(M)$ is residually simple.

A particular case of this is:

Corollary 1.3. Let $M$ be a finite volume hyperbolic 3-manifold. Then $\pi_{1}(M)$ is residually simple.

In fact we shall show more; the simple groups will all be of the type $\operatorname{PSL}(2, \mathbb{F})$ for finite fields $\mathbb{F}$ of prime cardinality. A corollary of Theorem 1.2 is a new proof of a result originally observed by Magnus, which follows by taking $M$ to be a handlebody:

Corollary 1.4. Nonabelian free groups are residually simple.

Received by the editors November 28, 1995.

1991 Mathematics Subject Classification. Primary 57M50, 57M60, 57R30.

Key words and phrases. 3-manifold, residually simple.

Supported by the NSF (both authors) and The Royal Society (second author).

(c)1998 American Mathematical Society 


\section{General REMARKS}

We start with some general remarks about algebraic representations of 3-manifold groups, and groups $P S L(2, \mathbb{F})$ which do not necessarily surject. This is just a reformulation of classical notions about linear groups; cf. [6]. For details on number fields and their completions, see [2] for instance.

Let $M$ be a compact orientable 3-manifold, and $\rho$ an algebraic representation of $\pi_{1}(M)$ into $S L(2, \mathbb{C})$. Denote the image of the group $\pi_{1}(M)$ under $\rho$ by $\Gamma$. Let $k=\mathbb{Q}(\operatorname{tr} \gamma: \gamma \in \Gamma)$ denote the trace-field of $\Gamma$. Since $\rho$ is an algebraic representation, $k$ is a finite extension of $\mathbb{Q}$. Let $A \Gamma$ be the algebra

$$
\left\{\sum a_{i} \gamma_{i}: a_{i} \in k, \gamma_{i} \in \Gamma\right\}
$$

This is a quaternion algebra over $k$ as follows from [1]. We remark that for finite volume hyperbolic 3-manifolds $k$ is always a finite extension of $\mathbb{Q}$; cf. [4], Proposition 6.7.4.

By the classification theorem for quaternion algebras $A \Gamma$ is unramified at all but a finite number of places of $k,[5]$. In particular, for all but a finite number of prime ideals $\wp$ of $k, A \otimes_{k} k_{\wp} \cong M\left(2, k_{\wp}\right)$. Thus on specifying an isomorphism between $A \otimes_{k} k_{\wp}$ and $M\left(2, k_{\wp}\right)$, we induce a representation of $\Gamma$ into $S L\left(2, k_{\wp}\right)$.

Since $M$ is compact, $\Gamma$ is finitely generated and finitely presented, therefore for all but finitely many prime ideals, we actually induce a representation of $\Gamma$ into $S L\left(2, O_{\wp}\right)$ where $O_{\wp}$ are the $\wp$-adic integers in $k_{\wp}$, since only finitely many $k$-primes can divide denominators of elements of $\Gamma$.

Denote by $\pi_{\wp}$ a local uniformizing parameter for $O_{\wp}$. The unique maximal ideal of $O_{\wp}$ is $\pi O_{\wp}$, and $O_{\wp} / \pi O_{\wp}$ is a finite field with $p^{n}$ elements where $\wp$ divides $p$ for a rational prime $p$ and $n$ is the inertial degree of $\wp$. Therefore reduction induces a homomorphism (so far, not necessarily surjective) of $\Gamma$ into $S L\left(2, \mathbb{F}_{p^{n}}\right)$ where $\mathbb{F}_{p^{n}}$ is the finite field with $p^{n}$ elements. By composing the map $\pi_{1}(M) \rightarrow \Gamma$, with the above, and then projectivising, we get a homomorphism $\phi_{\wp}$ of $\pi_{1}(M)$ into $P S L(2, \mathbb{F})$, for infinitely many finite fields $\mathbb{F}$.

Lemma 2.1. There are infinitely many $k$-primes $\wp$ such that the homomorphisms $\phi_{\wp}$ constructed above are nontrivial and map $\pi_{1}(M)$ into $P S L(2, \mathbb{F})$ where $\mathbb{F}$ has prime cardinality.

Proof. It is a well-known consequence of how prime ideals behave in finite extensions of $\mathbb{Q}$, that there are infinitely many rational primes that split completely in the finite extension $k / \mathbb{Q}$; see [2] Theorem 4.12 for example. Now a rational prime $p$ splits completely if and only if the inertial degree of the $k$-prime divisors of $p$ are all equal to 1 . In particular we deduce that there are infinitely many rational primes $p$ with $\wp \mid p$ such that $\phi_{\wp}$ maps $\pi_{1}(M)$ into $P S L\left(2, \mathbb{F}_{p}\right)$ where $\mathbb{F}_{p}$ has $p$ elements.

It is also easy to see that infinitely many of these homomorphisms are non-trivial. For if $\gamma \in \Gamma$ is given, reduction of, for example, the $(1,2)$-entry of $\gamma$ will be non-zero for all but a finite number of $k$-primes - since entries of $\gamma$ will be $\wp$-adic units for all but a finite number of $\wp$. Hence, the number of $k$-primes such that $\phi_{\wp}(\gamma)$ is trivial is finite.

In particular Lemma 2.1 implies that we may construct infinitely many nontrivial representations of $\pi_{1}(M)$ into groups $P S L(2, \mathbb{F})$ where the cardinalities of $\mathbb{F}$ are distinct primes. 


\section{Proof of Theorem 1.2}

To prove Theorem 1.2 we shall make use of the description of subgroups of the groups $\operatorname{PSL}(2, \mathbb{F})$ where $|\mathbb{F}|$ is of odd prime cardinality. The following is deduced from [3], Theorem 6.25, together with the observation that all abelian subgroups of such $\operatorname{PSL}(2, \mathbb{F})$ are cyclic.

Theorem 3.1. Let $p$ be an odd rational prime; then a complete list of subgroups of $\operatorname{PSL}(2, \mathbb{F})$ where $|\mathbb{F}|=p$ is

1. Cyclic groups of order $p$ and order $n$ where $n$ divides $\frac{(p \pm 1)}{2}$.

2. Dihedral groups of order $n$ where $n$ is as in 1 .

3. Semi-direct products of cyclic groups of order $p$ with cyclic groups of order $(p-1) / 2$.

4. $A_{4}, S_{4}$ or $A_{5}$.

We now show that the homomorphisms $\phi_{\wp}$ constructed above actually surject infinitely many groups $P S L(2, \mathbb{F})$ as in the statement of Lemma 2.1.

The group $\Gamma$ is never soluble of any finite degree. This follows for example by the fact that they contain free non-abelian groups, as they are non-elementary subgroups of $S L(2, \mathbb{C})$. Thus for the remainder of the proof, we fix some nontrivial element $\alpha$ which lies deep in the solubility series of $\pi_{1}(M)$.

Now suppose then that we are given some element $\gamma \in \Gamma$. As observed above, we can find (infinitely many) $k$-primes $\wp$ so that $\phi_{\wp}(\gamma) \neq 1$ and $\phi_{\wp}(\alpha) \neq 1$.

Since the homomorphic image of a term in the solubility series for a group lies in the same term of the solubility series of the image, the fact that the element $\alpha$ maps nontrivially means that the image of the group $\pi_{1}(M)$ cannot be of type 1 , 2, 3 nor $A_{4}$ or $S_{4}$ in the list provided by Theorem 3.1 since these are all soluble of small fixed degree.

Thus the map $\phi_{\wp}$ will be shown to have been a surjection if we show that $\phi_{\wp}(\Gamma) \neq$ $A_{5}$ for infinitely many $\wp$. Now if infinitely many of the homomorphisms constructed surject $A_{5}$, then since there are only finitely many normal subgroups in $\Gamma$ of index 60 , it follows that for infinitely many of these homomorphisms the kernels coincide. However this is impossible. These homomorphisms were constructed by reducing $\Gamma$ modulo $\pi_{\wp} O_{\wp}$, hence if infinitely many homomorphisms had the same kernel this would mean that the elements in this matrix group were congruent to the identity modulo infinitely many $\pi_{\wp} O_{\wp}$, which is clearly false.

Thus we have $\phi_{\wp}$ that surjects $\pi_{1}(M)$ onto some $P S L(2, \mathbb{F})$ and which maps $\gamma$ non-trivially.

In fact we may conclude that under the homomorphisms $\phi_{\wp}$ constructed above, $\pi_{1}(M)$ surjects infinitely many of the simple groups $P S L(2, \mathbb{F})$, with $|\mathbb{F}|$ of odd prime cardinality.

\section{Application}

A motivation for this result arises from trying to show that covers of hyperbolic 3 -manifolds have positive first Betti number. With this in mind, an application of this result stems from the following question raised by D. Cooper. Here $\operatorname{inj}(M)$ denotes the injectivity radius of $M$, which is simply half the length of the shortest closed geodesic in $M$ :

Question. Is there a number $K>0$ so that if $M$ is a closed hyperbolic 3-manifold and $\operatorname{inj}(M)>K$, then $\operatorname{rank}\left(H_{1}(M ; \mathbb{Q})\right)>0$ ? 
An affirmative answer to this question, taken with the fact that $\pi_{1}(M)$ is residually finite, implies in particular that every closed hyperbolic 3-manifold has a finite sheeted covering with positive first Betti number. However our main theorem shows that actually more would be true.

Corollary 4.1. If the above question has an affirmative answer, then every rational hyperbolic homology 3-sphere has infinite virtual Betti number.

Proof. We recall that $M$ is said to have infinite virtual Betti number if given any integer $N$, one can find a finite sheeted covering of $M$ whose first Betti number is larger than $N$. Equivalently, $M$ has infinite virtual Betti number if the rank of $H_{2}(\widetilde{M} ; \mathbb{Z})$ is unbounded as $\widetilde{M}$ ranges over all finite covers of $M$.

Since, given any constant $C, M$ has only finitely many geodesics of length at most $C$, our main theorem implies that one can find regular covers of $M$ having arbitrarily large injectivity radius for which the group of covering transformations has the form $\operatorname{PSL}(2, \mathbb{F})$. An affirmative answer to the question implies that we may assume that these manifolds all have $H_{2}\left(M_{\mathbb{F}} ; \mathbb{Z}\right)$ having rank at least one. The action of the covering group gives a series of representations

$$
\alpha_{\mathbb{F}}: P S L(2, \mathbb{F}) \longrightarrow G L\left(H_{2}\left(M_{\mathbb{F}} ; \mathbb{Z}\right)\right)
$$

Suppose to the contrary that the ranks of the groups $H_{2}\left(M_{\mathbb{F}} ; \mathbb{Z}\right)$ were bounded, by $P$ say. Then since there is a bound on the size of the finite subgroups in $G L(P, \mathbb{Z})$, and the sizes of the groups $P S L(2, \mathbb{F})$ are going to infinity, we would eventually see that some $\alpha_{\mathbb{F}}$ is nonfaithful, hence trivial. It follows that the fixed homology

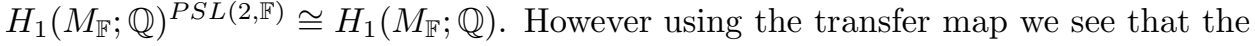
left hand side of this isomorphism is $H_{1}(M ; \mathbb{Q})$, a contradiction, since we assumed that $M$ was a rational homology sphere.

\section{REFERENCES}

1. H. Bass, Groups of integral representation type, Pacific J. Math. 86 (1980), 15-51. MR 82c: 20014

2. W. Narkiewicz, Algebraic Numbers, Polish Scientific Publishers, Warsaw, 1974. MR 50:268

3. M. Suzuki, Group Theory I, Grundlehren der math. Wissen. 247, Springer-Verlag, 1980. MR 82k:20001c

4. W. P. Thurston, The Geometry and Topology of 3-Manifolds, Mimeographed lecture notes, Princeton University, 1977.

5. M-F. Vignéras, Arithmétique des algèbres de quaternions. L. N. M. 800, Springer-Verlag 1980. MR 82i: 12016

6. B. A. F. Wehrfritz, Infinite Linear Groups, Ergeb. Math. Grenz. 76, Springer-Verlag, 1973. MR 49:436

Department of Mathematics, University of California, Santa Barbara, California 93106

E-mail address: long@math.ucsb.edu

Department of Mathematics, University of Texas, Austin, Texas 78712

E-mail address: areid@math.utexas.edu 